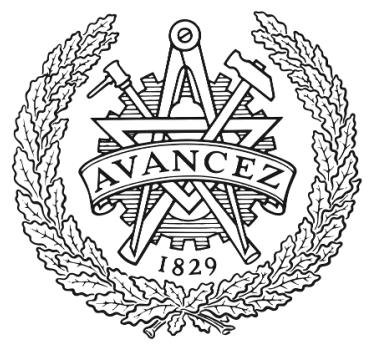

CHALMERS

UNIVERSITY OF TECHNOLOGY

\title{
Dual-Comb Swept Wavelength Interferometry
}

Downloaded from: https://research.chalmers.se, 2023-04-26 09:47 UTC

Citation for the original published paper (version of record):

Mazur, M., Fontaine, N., Karlsson, M. et al (2020). Dual-Comb Swept Wavelength Interferometry. Conference Proceedings - Lasers and Electro-Optics Society Annual Meeting-LEOS, 2020-May. http://dx.doi.org/10.1364/CLEO_AT.2020.JW2B.23

N.B. When citing this work, cite the original published paper. 


\title{
Dual-Comb Swept Wavelength Interferometry
}

\author{
Mikael Mazur $^{(1,2)}$, Nicolas K. Fontaine ${ }^{(2)}$, Magnus Karlsson ${ }^{(1)}$, Peter A. Andrekson ${ }^{(1)}$, Victor \\ Torres-Company ${ }^{(1)}$ and Jochen Schröder ${ }^{(1)}$ \\ (1) Photonics Laboratory, Department of Microtechnology and Nanoscience, Chalmers University of Technology, Gothenburg, Sweden \\ (2) Nokia Bell Labs, Crawford Hill Lab, 791 Holmdel Rd, Holmdel, 07733, NJ, USA \\ mikael.mazur@chalmers.se,jochen.schroeder@chalmers.se
}

\begin{abstract}
We demonstrate a hybrid swept wavelength interferometry - dual-comb spectroscopy system for fast broadband full-field measurements. Phase and amplitude retrieval is experimentally verified and compensation of laser sweep nonlinearity is demonstrated without external referencing.
\end{abstract}

\section{Introduction}

Swept wavelength interferometry (SWI) [1] and dual-comb spectroscopy (DCS) [2,3] are two key methods for precision measurements used in applications such as ranging, molecular spectroscopy and as generic tools for device under test (DUT) characterization. SWI uses a frequency sweeping laser, as shown in Fig. 1(a), and therefore achieves high signal-to-noise ratio (SNR). However, broadband measurements require long sweeps over which the DUT must be stable. Moreover deviations from a purely linear wavelength sweep cause significant measurement errors which requires additional reference interferometers or other external referencing for compensation. This makes accurate measurements of small group delay variations and higher-order dispersion, challenging. In DCS, two frequency combs with a small difference in spacing are used, as shown in Fig. 1(b). DCS enables accurate measurements of wavelengthdependent, broadband, phase and amplitude transfer functions. However, the discrete nature of the combs implies that narrow-band variations that fall between comb lines, or delays that correspond to more than the frequency spacing of the combs, cannot be measured. Comb spacing is therefore an SNR and spectral sampling trade-off.

Here we present a hybrid method that combines the principles of SWI and DCS by using a tunable laser source for DCS. Sweeping the source laser over the $25 \mathrm{GHz}$ comb spacing enables fast, high precision, single shot measurements over a bandwidth of $1.1 \mathrm{THz}$ with a resolution of $50 \mathrm{kHz}$, corresponding to minimum/maximum measurable delays of $0.9 \mathrm{ps}$ and $20 \mu \mathrm{s}$. In addition, by using information from multiple measured comb lines, the nonlinearities of the laser sweep can be tracked and compensated for without any external reference interferometer. We experimentally demonstrate amplitude and phase retrieval of several prototype device under tests to show the flexibility of our approach, highlighting the potential for metrological applications.

\section{Measurement Principle and Experimental Setup}

In contrast to using a fixed highly coherent seed laser and tuning the comb spacing for superresolved DCS [4] or using multiple seed lasers [5], the proposed system keeps the comb spacing fixed and sweeps the seed laser to simultaneously measure the intermediate frequencies over the full bandwidth. This has the key benefit of being agnostic to comb spacing, enabling combs with e.g. THz-spacing to be used, which is not possible using previous methods. It also opens the possibility to use different comb platforms such as integrated electro-optic combs and tunable microring resonator combs [6]. Here, we used two electro-optic frequency combs, generated with two cascaded phase modulators and an intensity modulator. The combs generated about 45 lines with 25 and $25.1 \mathrm{GHz}$ line spacing, respectively, and were seeded by a sweeping laser source $(40 \mathrm{~nm} / \mathrm{s})$, as shown in Fig. 1(c). The laser is swept $26 \mathrm{GHz}$, and the measured electrical signal contains multiple parallel SWI-like scans, each being present at different beat notes separated by the
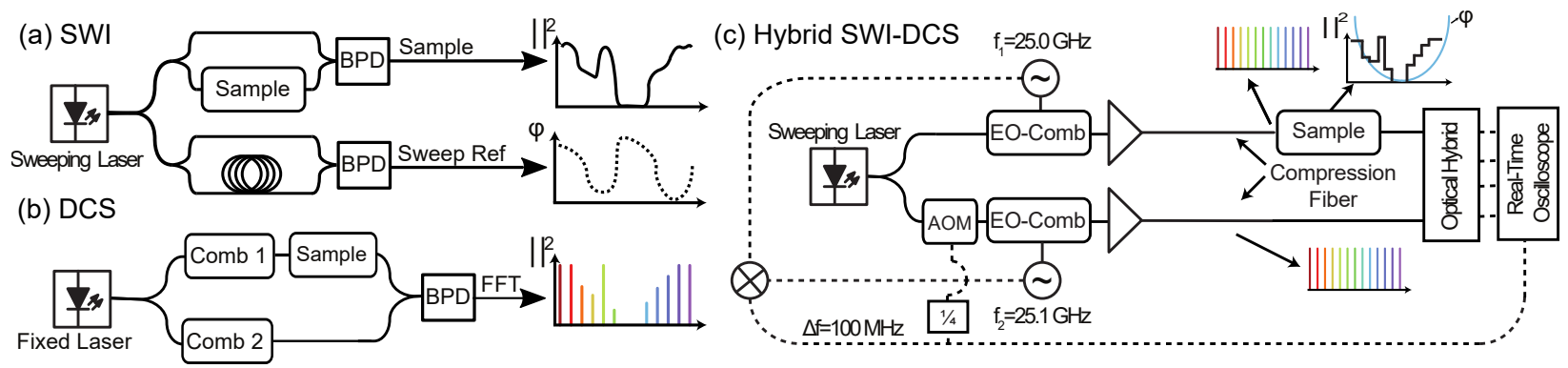

Fig. 1: (a) Swept wavelength interferometry measurements using an additional interferometer to measure laser sweep nonlinearities. (b) Dual comb spectroscopy using two combs with a small line spacing difference. (c) Experimental setup for the proposed hybrid system combining SWI and DCI for fast broadband full-field measurements. (EO: Electro-optic, BPD: Balanced Photo Detector, AOM: Acousto-Optic Modulator) 
(a)
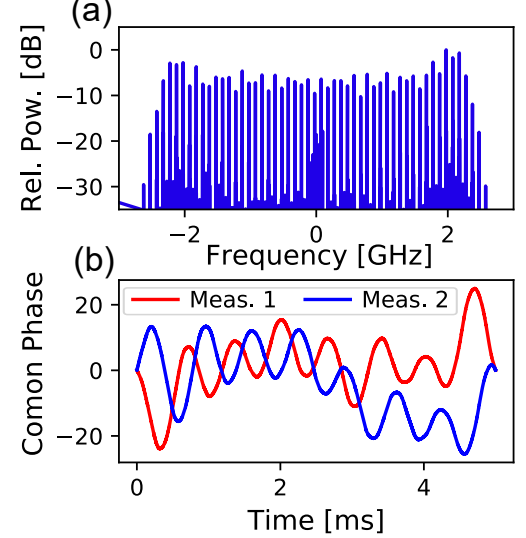

(c)

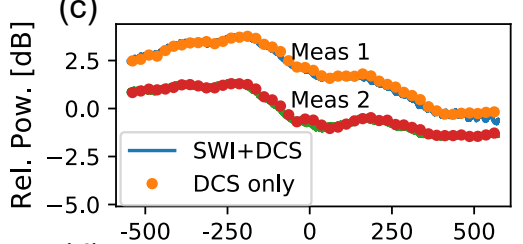

(d)

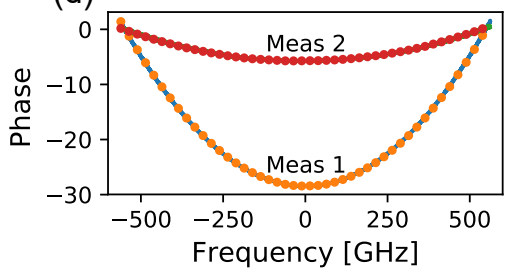

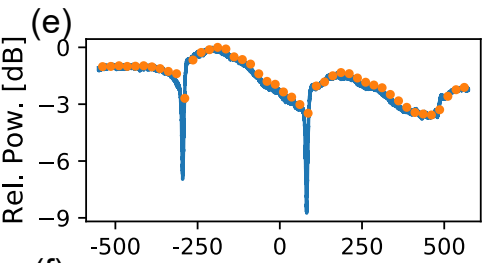

(f)

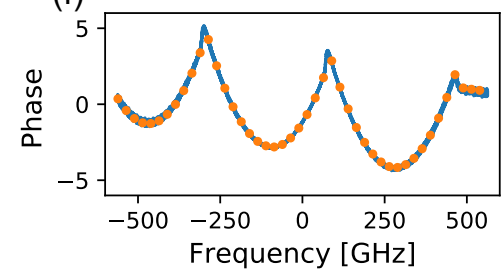

Fig. 2: (a) Fourier-transform of the measured electrical signal displaying the $100 \mathrm{MHz}$-spaced RF comb. (b) Recovered common phase from all comb lines showing the nonlinear laser sweep from two independent measurements. (c) Recovered amplitude and (d) recovered phase for two measurements of applied quadratic dispersion. (e) Recovered amplitude and (f) phase for an arbitrary phase profile with two $10 \mathrm{GHz}$ notch filters. Note that the notches are not visible using only DCS with the $25 \mathrm{GHz}$-spaced combs.

relative comb spacing, similar to the standard DCS setup in Fig. 1(b). An optical $90^{\circ}$-hybrid followed by a $6.25 \mathrm{GS} / \mathrm{s}$ real-time oscilloscope digitized the signal. The sampling clock was locked to the $100 \mathrm{MHz}$ beating signal from the combs' clocks, as shown in Fig. 1(c).

The processing consists of multiple steps, resembling a mixture of signal processing used for SWI and DCI, respectively. Fourier-transforming the raw measured electrical signal gives the resulting RF-comb, as shown in Fig. 2(a). The scan of each comb-line is selected by bandpass filtering, producing 45 parallel SWI scans. By using post processing similar to windowing in SWI at this stage [7], flexible trade-off between SNR and spectral resolution resolution is possible. The measured phase is then averaged over all lines to extract the nonlinear laser sweep, as shown in Fig. 2(b). The measured traces resemble well previous measurements with equivalent lasers [7]. The averaging accounts for power variations between lines to accurately estimate the common phase. A separate reference interferometer is therefore not needed. After compensating the laser sweep, calibration of each line with respect to previous reference measurement is performed. Finally, via the overlap regions, the traces are stitched to produce the broadband full-field measurement.

\section{Results and Conclusions}

To verify the proposed method, we performed three proof-of-principle experiments using a programmable wavelength selective switch (WSS) as the DUT. First, two different quadratic dispersion profiles were applied and measured, as shown in Fig. 2(c) and (d). Corresponding measurements using conventional DCS (orange dots) show excellent agreement. Amplitude variations arising from the WSS are clearly visible in Fig. 2(c). To furthermore demonstrate the power of the proposed technique, we programmed a general phase profile together with two amplitude notch filters, each $10 \mathrm{GHz}$ wide. The resulting measurements are shown in Fig. 2(e) and (f) for amplitude and phase response, respectively. Here we clearly observe the power of the proposed method by enabling high precision measurement in the intermediate regime between DCS and SWI. For DCS, the notches are not visible as the bandwidth is narrower than the comb line spacing, but with the proposed technique they can easily be distinguished. The programmed notch depth was 8 and $10 \mathrm{~dB}$ of attenuation, respectively, showing good agreement with the results in Fig. 2(e). Similarly, the retrieved phase shows a very good match between the proposed system and DCS measurements, with the proposed system capable of resolving intermediate spectral features.

In conclusion, we have presented a system based on a combination of swept wavelength interferometry and dualcomb spectroscopy. By combining broadband frequency combs and sweeping over the line spacing, fast and accurate full-field measurements can be performed. High resolution phase and amplitude retrieval and compensation of the laser sweep nonlinearities without external reference interferometer is experimentally demonstrated.

Acknowledgement This work was financially supported by the Knut and Alice Wallenberg Foundation (KAW).

\section{References}

[1] G. D. VanWiggeren and D. M. Baney, "Swept-wavelength interferometric analysis of multiport components", IEEE PTL, 15 (9) (2003)

[2] I. Coddington et al.,"Dual-comb spectroscopy", Optica, 3 (4) (2016)

[3] V. Durán et al., "Ultrafast electrooptic dual-comb interferometry", Opt. Express 23 (23) (2015)

[4] S. Wang et al., 'Fast MHz spectral-resolution dual-comb spectroscopy with electro-optic modulators", Optics Letters, 44 (1) (2019)

[5] T. Nishikawa et al., "Broadband Dual-comb Spectroscopy with Cascaded-electro-optic-modulator-based...", Proc. CLEO paper. SW3G.2 (2015)

[6] T. Lin et al.,"Broadband High-Resolution Scanning of Soliton Micro-Combs ”, Proc. CLEO paper SF3H.6 (2019)

[7] E. D. Moore, "Advances in Swept-Wavelength Interferometry for Precision Measurements", Graduate Theses \& Dissertations. 18., (2011) 